\title{
Organized recall: Individual functions
}

GEORGE MANDLER, University of California, San Diego, La Jolla, Calif. 92037

Individual Ss' recall of categorized lists is shown to vary systematically with number of categories used, adding about four words per category. The recall performance of an individual $S$ can be manipulated and reliable individual Category-Recall functions were obtained. The study supports previous theoretical work on long-term memory and suggests further approaches to the study of individual differences in recall.

The dependence of free recall on organization has been explored extensively during the past decade. Previous studies (Mandler, 1967) have shown that the recall of lists of unrelated words is a linear function of the number of categories used in organizing the lists. In a typical experiment, Ss are run individually and required to sort a list of 100 words into anywhere from two to seven categories, with Ss choosing the number of categories. This sorting process is repeated until stable categorization (i.e., $100 \%$ or $95 \%$ identity of two consecutive sorting trials) is achieved. Immediately following this organization of the list the $S$ is required to recall as many of the words on the original list as possible. The experiments showed a highly significant linear relationship between the number of categories (NC) used during the sorting and the number of items recalled (R). The median slope for this relationship was 3.9; the median correlation between NC and R was .70. Thus Ss added about four words to recall for every additional category used in organization. The model suggested (Mandler, 1967, 1968) was one where Ss recall categories and approximately $5 \pm 2$ words per category.

One possible artifact in these results involves a hidden trial or time effect such that Ss using low NC used few trials and therefore recalled less. However, the NC-R relationship held even when total time and trials were held statistically and experimentally constant. Another possible artifact, that of self-selection, i.e., that Ss using few categories were also poor on the memory task, was discarded when the NC-R relationship held even when Ss were instructed on the number of categories they were to use.

All previous experiments demonstrated the NC-R relationship in a between-S design, with different Ss providing the different points in the NC-R relation. If the model holds, the same relationship should hold when a single $S$ provides all the points for the NC-R relation. Thus, a S's ability to recall material from a list of words should be primarily dependent on how he organizes that list, i.e., how many categories he uses, and not on some constant memory ability or other variable. While the enforced use of a specified number of categories may not enable Ss to use the organization fully, the slope of the NC-R functions should be close to the value of 3.9 obtained in the between-S design.

\section{METHOD}

Five Ss were used to obtain individual NC-R functions. Each $\mathrm{S}$ was run for 12 individual sessions except for $\mathrm{S} 3$ who was given two sets of 12 sessions. Four of the Ss were college undergraduates and one was a high school senior. All were paid for their services.

Twelve lists of 100 words were constructed by selecting 1200 nouns from the Thorndike-Lorge (1944) word list with a count of 40 per million or higher. The 12 lists were randomly selected from this pool.

Each S was given two sessions at each NC level from 2 to 7 . The first six sessions were a random order of the six levels, and this order was repeated for the second six sessions. Each $\mathrm{S}$ was given two sessions per week, three to four days apart.

In each session a $S$ was given one of the 12 sets of 100 words, printed on $3 \times 5$ index cards. According to the schedule for that particular session, he was asked to sort these 100 words into two to seven categories. He was told to use any categories, rules, or concepts he wished, by simply putting words together that belonged together, but not to sort on an alphabetic basis or on physical word characteristics. Following the first sort, the cards were repeatedly shuffled and he was instructed to sort them again into the same number of categories. The $\mathrm{S}$ was also told that he would continue this process until he had sorted the words in the same way on two consecutive sorting trials. In fact-based on past experimental evidence-we terminated the sorting task when $S$ had sorted to a $95 \%$ criterion of consecutive stability. Following the second criterion trial, the $\mathrm{S}$ was given a sheet of paper and asked to write down all the words from the list of 100 that he could recall. Thus, each $S$ produced two recall points $(R)$ for each level of categorization (NC) over a period of six weeks.

\section{RESULTS}

The results are shown in Fig. 1. The points plotted are in all cases the mean of the two observations for the NC values. For each $S$, we have also plotted the line of best fit obtained by the method of least squares, and have shown the formula for that line. Product moment correlations for the 12 points were also computed. S 3 was run twice on the identical procedure with the second set of 12 sessions separated from the first by a six-week period. The 12 sets of words used for his second set was a new randomly selected set of 12 sets of 100 words drawn from the original pool. His first run is shown with solid lines and circles, his second by broken ones.

The data clearly show that the NC-R relation can be obtained in the within-S design. Individual Ss' recall of organized lists of words varies directly with categorization.

Early in the experimental sessions, it was noted that S 3-contrary to past experience with some $200 \mathrm{Ss}-$-repeatedly used many categories of Size 1 in sorting the word lists. Since the theory demands and previous data suggest that Ss recall no less than approximately four words from each category, the performance of this $S$ with a maximum recall of one from categories of that size could not possibly have been within expected bounds. However, his first 12 sessions were completed to determine whether his data would produce an attenuated NC-R function. Final analysis showed that of the 54 categories produced by this S, 30\% had only one word in them, and $56 \%$ had less than five words. During his second set of 12 sessions, S 3 was instructed to put no less than five words into each category to determine whether his recall performance could be brought within the normal limits.

As expected, the slope for S 3 on Set 1 is low at .74, but even that slight relationship is statistically significant, $\mathrm{r}=.49$ $(p<.05)$. For the other four Ss and for S 3's second session, the five slopes have a median of 3.60 , essentially replicating the between-S data. The five correlations range from .69 to .76 $(p<.01)$. Since S 3, during his second session, was told to use only five words per category, his slope did not quite come up to the value of the other four Ss, who consistently used larger categories.

The intercepts for the five Ss are more variable, ranging from 8.74 to 25.93 . Of the two sets of values, the intercept represents probably the more appropriate measure of individual differences. Mandler (1967) has suggested that the intercept represents recall based on organization other than that expressed by the categories used during sorting. Such 

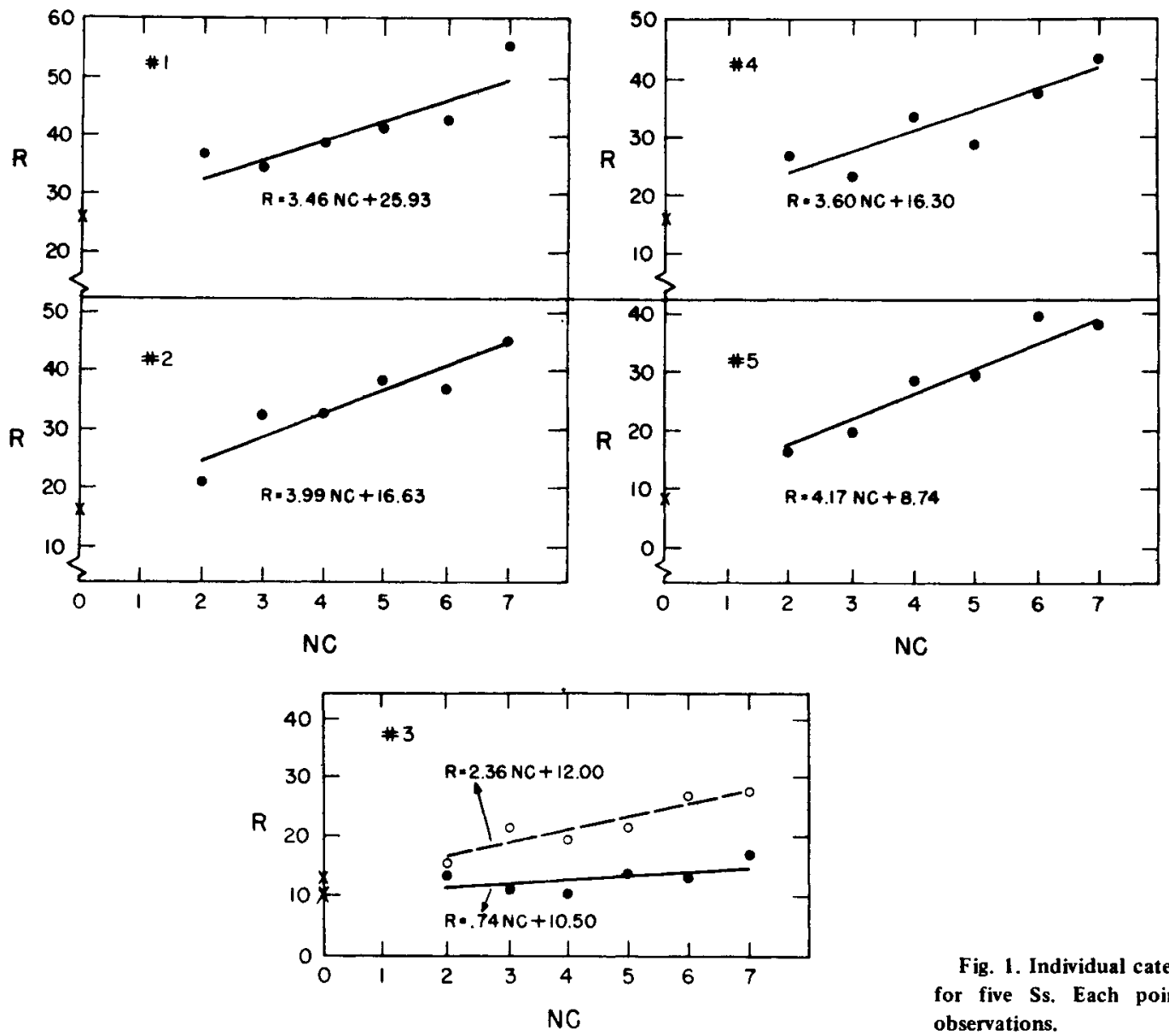

Fig. 1. Individual category-recall (NC-R) functions for five Ss. Each point represents mean of two observations.

extratexperimental organization not only affects the intercept of the line of best fit but also reflects individual differences in the way material is organized. The slope on the other hand reflects a basic limitation of human memory and should. on the average, show little individual variation.

\section{DISCUSSION}

Previous research on individual differences in human memory has focused on empirical measures. essentially unrelated to theories of human memory. The present demonstration shows that individual recall concords with a general theoretical point of view. It is highly sensitive to organizational factors. and (as in the case of $S 3$ ) the general level of performance can be altered by shifts in organizational strategies.

\section{REFERENCES}

MANDLER, G. Organization and memory. In $K$. W. Spence and J. T. Spence (Eds.), Psychology of learning and motivation, Vol. 1. New York: Academic Press, 1967.

MANDLER, G. Association and organization: Facts, fancies, and theories. In T. R. Dixon and D. L. Horton (Eds.), Verbal behavior and general behavior theory. Englewood Cliffs, N.J.: Prentice Hall, 1968.

THORNDIKE, E. L., \& LORGE, I. The teacher's word book of 30.000 words. New York: Teachers College, Columbia University, 1944.

\section{NOTE}

1. Supported in part by NSF Grant GB-7807. 1 wish to thank Barbara Cragin and Zena Pearlstone for technical assistance. 